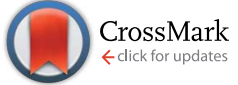

Cite this: RSC Adv., 2017, 7, 7780
Received 23rd November 2016 Accepted 14th January 2017

DOI: 10.1039/c6ra27198g

www.rsc.org/advances

\section{Evaluation of antioxidant, anti-inflammatory and anti-proliferative activities of ethanol extracts from different varieties of Sanghuang species}

\author{
Wang-Ching Lin, $\dagger^{a}$ Jeng-Shyan Deng, $\dagger^{\mathrm{b}}$ Shyh-Shyun Huang, ${ }^{a}$ Sheng-Hua Wu, ${ }^{c}$ \\ Hui-Yi Lin*a and Guan-Jhong Huang*d
}

Sanghuangporus sanghuang (S. sanghuang) is a rare fungal species that grows on wild mulberry trees, and its yield is much lower than that of valuable medicinal herbs such as saffron and other fungal species such as Cordyceps sinensis and Antrodia cinnamomea. The annual production of Sanghuangporus vaninii (S. vaninii) is approximately 40 tons. Sanghuangporus baumii (S. baumii) is widely distributed and commonly used. Most studies so far have focused on Tropicoporus linteus (T. linteus). These three Sanghuang varieties (T. linteus, S. vaninii, S. baumii) are currently considered as having the best pharmacological efficacy. Until now, no reports have demonstrated that $S$ sanghuang has the same efficacy as that of $T$. linteus and S. baumii. The objective of this study was to analyze the pharmacological activity of the wild medicinal fungus S. sanghuang (on Morus) in comparison with that of other commonly available Sanghuang varieties. The results of this study provide a basis for future use of these Sanghuang species as dietary supplements or therapeutic agents. We evaluated the cancer cell inhibition, free radical scavenging ability, and anti-inflammatory effects of the three Sanghuang varieties. The results showed $S$. sanghuang to be the most efficacious Sanghuang basidiocarp of the two varieties, rich in polyphenolic compounds, having antioxidant, anti-inflammatory, and tumor suppressive ability, and indicating a possible use of this variety as dietary supplements or natural herbal medicine in the future.

\section{Introduction}

If there are unpaired electrons present, an atom or molecule will be unstable and become an active free radical. In the human body, free radicals, which are responsible for the immune system and inflammatory responses under normal conditions, are mainly produced by various enzyme systems, activated neutrophil leucocytes, and ischemic mitochondria., ${ }^{\mathbf{1 , 2}}$ When present in large quantities, free radicals can react with intracellular lipids, proteins, nucleic acids, and signal transduction factors via redox reactions, causing other molecules to become unstable and damage human cells. ${ }^{1-3}$ The human body has a corresponding antioxidant mechanism to lower the toxicity of redundant free radicals. ${ }^{3}$ If the number of free radicals exceeds the antioxidant

${ }^{a}$ School of Pharmacy, China Medical University, Taichung 404, Taiwan. E-mail: linwc0913@gmail.com; sshuang@mail.cmu.edu.tw; hylin@mail.cmu.edu.tw; Tel: +886-4-2205-3366 ext. 5512

${ }^{b}$ Department of Health and Nutrition Biotechnology, Asia University, Taichung 413, Taiwan. E-mail: dengjs@asia.edu.tw

${ }^{c}$ Department of Biology, National Museum of Natural Science, Taichung 404, Taiwan. E-mail: shwu@mail.nmns.edu.tw

${ }^{d}$ Department of Chinese Pharmaceutical Sciences and Chinese Medicine Resources, College of Pharmacy, China Medical University, Taichung 404, Taiwan. E-mail: gjhuang@mail.cmu.edu.tw; Tel: +886-4-2205-3366 ext.5508

$\dagger$ These authors contributed equally to this work. ability, oxidative stress occurs. ${ }^{3,4}$ Currently, oxidative stress is known to damage the human body and is involved in aging, cancer, asthma, and inflammatory responses. ${ }^{4-7}$

The invasion of bacteria or viruses can cause immune responses in the human body. ${ }^{8}$ As a result, macrophagocytes are activated to withstand foreign pathogens and mitigate damage. ${ }^{\mathbf{8} 9}$ Excessive inflammatory responses, however, cannot only cause septicemia but are also closely related to many other diseases such as autoimmune diseases and cancer. ${ }^{10}$ Endotoxin is a polysaccharide secreted from Gram-negative bacteria endotoxin invasion stimulates macrophagocytes to produce various inflammatory regulators such as nitric oxide, leukotrienes, prostaglandins, interleukins, and tumor necrosis factor (TNF)- $\alpha$. Among the regulators, interleukin (IL)-1, IL-6, and TNF$\alpha$ play a significant role in acute inflammatory responses. ${ }^{\mathbf{1 1 - 1 3}}$ These three cytohormones can increase vasopermeability, resulting in inflammatory responses such as redness, swelling, fever, and aches. They can also continuously stimulate the activation of macrophagocytes and secrete additional proinflammatory cytokines. ${ }^{\mathbf{1 4}}$ Thus, if the production of inflammatory media can be decreased, the symptoms of inflammation can be attenuated.

In recent years, cancer has been one of the most important causes of death worldwide. Currently, studies have focused on the discovery or development of medicines that can selectively 
induce tumor cells to undergo apoptosis but are nontoxic to normal cells. ${ }^{15}$ Introduction of medicine to induce tumor cells for apoptosis has relatively lower side effects, compared to using traditional medicine to cause cell necrosis. ${ }^{\mathbf{1 5 , 1 6}}$ Numerous recent studies have identified many effective components in natural Chinese herbal medicines that can effectively treat malignant tumors; these effective components were found to have significant anticancer activities. ${ }^{17}$ For instance, taxol and curcumin were found to be efficacious in restraining tumor cell growth. ${ }^{18}$ The use of the effective components in natural Chinese herbal medicine to control the growth of tumor cells and induce their apoptosis is an attractive research target in the field of medicine selection and is an important topic in current research.

The medicinal value of fungus Sanghuang has been known in China, Japan, and Korea for more than a thousand years. In early 2012, a new species of the fungus was identified by Wu et al., named Sanghuangporus sanghuang. ${ }^{19,20}$ Sanghuang species grow in a very limited number on wild mulberry trees and are found to be distributed in mainland China, Japan, Korea, and Taiwan. ${ }^{19-21}$

Recent studies have shown that Sanghuang (Tropicoporus linteus or Sanghuangporus baumii) exhibits good pharmacological activities such as immunity enhancement; anti-tumor, antibacterial, anti-inflammatory, and antioxidant activities; blood sugar regulation, hepatoprotection; and prevention of stomach ulcer. ${ }^{21-24}$ According to the literature, Sanghuang (T. linteus or $S$. baumii) is rich in active components including polyphenols, pyrans, furanones, triterpenoids, and polysaccharides. ${ }^{21,25}$

Currently, two species of Sanghuang are available in the Chinese traditional medicine market, namely Phellinus linteus, now known as T. linteus; $P$. baumii, now known as $S$. baumii. Most studies so far have focused on T. linteus. In addition, $P$. vaninii, now known as $S$. vaninii, a parasitic pathogen on the trunks of Populus davidiana, has a basidiocarp appearance very similar to that of the wild Sanghuang. ${ }^{19-21,26}$ Owing to its high yield in the wild and ease of artificial cultivation; it is sold as real Sanghuang in the markets in China, Japan, and Korea. S. baumii which has a very high yield of over hundreds of tons per year has also been extensively studied. However, owing to its lower efficacy when compared with that of the other three varieties $(T$. linteus, $S$. baumii, S. vaninii), recent years have seen a decline in its research. Studies on the other varieties are even rarer. ${ }^{19-21,26}$

Thus, in the past decade, T. linteus and $S$. baumii, but not the wild $S$. sanghuang, have been generally used as the scientific names for Sanghuang ( $S$. sanghuang) species and studied worldwide. $^{21}$ Till date, there have been no reports on any beneficial pharmacological activity of $S$. sanghuang.

In addition, a review of the existing studies found that most researchers believe that the pharmacological activity of T. linteus may be derived from its rich active components, such as hispidin or its derivatives and polyphenols. To date, there have been no studies indicating that $S$. sanghuang is also rich in the same components. Therefore, in our study, we selected phenolic compounds that are commonly found in T. linteus and hispidin derivatives obtained via isolation and purification as indicative components to investigate whether $S$. sanghuang also has similar active components and abundances for subsequent relevant studies.
Hence, the objective of this study was to examine the pharmacological activity of the wild medicinal fungus Sanghuang ( $S$. sanghuang, abbreviated in this article as SS), including evaluations of active markers, antioxidant capacity, anti-proliferative effect on tumor cells, and anti-inflammatory activity. Its pharmacological activity was compared with that of $S$. baumii (abbreviated in this article as SB) and S. vaninii (abbreviated in this article as SV), which are widely available in the market and used either in combination with or mistakenly as Sanghuang. This study provides a basis for further developing Sanghuang as a dietary supplement or a therapeutic agent.

\section{Materials and methods}

\subsection{Source of material}

This study used basidiocarps from two similar Sanghuang varieties: $S$. baumii (SB) grown on Syringa; $S$. vaninii (SV) grown on Populus and Sanghuang: S. sanghuang (SS) grown on Morus. Dr Sheng-Hua Wu of the Department of Botany of the National Museum of Natural Science, Taiwan kindly identified and supplied all three varieties.

\subsection{Sample extraction}

To obtain sample extracts from three Sanghuang varieties, dried Sanghuang powders were immersed in and extracted with $70 \%$ ethanol for five days, followed by filtration. Filtrates were concentrated under reduced pressure to remove ethanol. This step was repeated four times to obtain the extracts, which were then stored for subsequent analysis.

\subsection{Quantitative analysis by HPLC}

The analysis will be performed on a HITACHI HPLC L-5000 system equipped with adegasser, pumps, and a photodiode array detector linked to a PC computer running the software program HPLC LACHROM. For HPLC analysis, an aliquot (10 $\mu \mathrm{L})$ is injected into the columns and eluted at $40{ }^{\circ} \mathrm{C}$. The analytical column $(250 \times 4.6 \mathrm{~mm}$ i.d., $5 \mu \mathrm{m})$ used is Thermo Hypersil GOLD $\mathrm{C}_{18}$ (USA), and the detection wavelength. For photodiode array detection, the wavelengths of standard compounds at their respective maximum absorbance wavelength can monitored at the same time. Identification is based on retention times and on-line spectral data in comparison with authentic standards.

2.3.1. Determination of phenolic compounds. ${ }^{27}$ The mobile phase contained acidified water with acetic acid $(2.5 \%$, solvent A) and methanol (solvent B). The gradient program started with $10 \%$ solvent B for $0 \mathrm{~min}$, then linearly increased to $20 \%$ solvent B for another $5 \mathrm{~min}$. This linear gradient was followed by an isocratic elution until $30 \mathrm{~min}$ and reconditioning steps to return to the initial mobile phase condition. The flow rate was $0.8 \mathrm{~mL} \mathrm{~min}^{-1}$, and the injection volumes of standards and samples were $10 \mu \mathrm{L}$. Identification was based on retention times and photo diode array (PDA) spectra by comparison with commercial standards at $280 \mathrm{~nm}$.

2.3.2. Determination of hispidin derivative compounds. The mobile phase contained acidified water with acetic acid 
(2.5\%, solvent A) and acetonitrile (solvent B). The gradient program started with $10 \%$ solvent B for $0 \mathrm{~min}$, then linearly increased to $70 \%$ solvent B for another $55 \mathrm{~min}$. This linear gradient was followed by an isocratic elution until $60 \mathrm{~min}$ and reconditioning steps to return to the initial mobile phase condition. The flow rate was $1.0 \mathrm{~mL} \mathrm{~min}^{-1}$, and the injection volumes of standards and samples were $10 \mu \mathrm{L}$. Identification was based on retention times and photo diode array (PDA) spectra by comparison with commercial standards at $295 \mathrm{~nm}$.

\subsection{Total polyphenol content and antioxidant activities of crude extracts in vitro}

2.4.1. Determination of total polyphenol content. The total polyphenol contents of crude extracts were determined according to the method of Huang et al. (2013). ${ }^{27}$ Each extract of $20 \mu \mathrm{L}$ was added to $200 \mu \mathrm{L}$ distilled water and $40 \mu \mathrm{L}$ of FolinCiocalteu reagent. The mixture was allowed to stand at room temperature for $5 \mathrm{~min}$ and then $40 \mu \mathrm{L}$ of $20 \%$ sodium carbonate was added to the mixture. The resulting blue complex was then measured at $680 \mathrm{~nm}$. Gallic acid was used as a standard for the calibration curve. The polyphenol content was calibrated using the linear equation based on the calibration curve. The total polyphenol content was expressed as mg gallic acid equivalence (GE)/mg extract weight.

2.4.2. Determination of antioxidant activity by $\mathrm{ABTS}^{+}$ scavenging ability. The $\mathrm{ABTS}^{+}$scavenging ability was determined according to the method of Huang et al. (2013). ${ }^{27}$ Aqueous solution of ABTS ( $7 \mathrm{mM}$ ) was oxidized with potassium peroxodisulfate $(2.45 \mathrm{mM})$ for $12 \mathrm{~h}$ in the dark at room temperature. The $\mathrm{ABTS}^{+}$solution was diluted with $95 \%$ ethanol to an absorbance of $0.75 \pm 0.05$ at $734 \mathrm{~nm}$ (Beckman UV-vis spectrophotometer, Model DU640B). An aliquot $(20 \mu \mathrm{L})$ of each sample was mixed with $180 \mu \mathrm{L} \mathrm{ABTS}^{+}$solution and the absorbance was read at $734 \mathrm{~nm}$ after $1 \mathrm{~min}$.

2.4.3. Determination of antioxidant activity by DPPH radical scavenging ability. The effects of crude extracts on DPPH radicals were estimated according to the method of Huang et al. (2013). ${ }^{27}$ Aliquot $(20 \mu \mathrm{L})$ of crude extracts at various concentrations were each mixed with $100 \mathrm{mM}$ Tris-HCl buffer $(80 \mu \mathrm{L}, \mathrm{pH}$ 7.4) and then with $100 \mu \mathrm{L}$ of DPPH in ethanol to a final concentration of $250 \mu \mathrm{M}$. The mixture was shaken vigorously and left to stand at room temperature for $20 \mathrm{~min}$ in the dark. The absorbance of the reaction solution was measured spectrophotometrically at $517 \mathrm{~nm}$. The percentages of DPPH decolorization of the samples were calculated according to the equation: $\%$ decolorization $=[1-($ ABS sample/ABS control $)] \times$ 100 (ABS: absorbance value).

\subsection{Determination of anti-inflammatory by mouse cell lines}

2.5.1. Cell cultumouse cell lines. For the experiment, a mouse macrophage RAW 264.7 cell line was used, which was obtained from the Food Industry Research and Development Institute. Cells were cultured in Dulbecco's Modified Eagle's Medium (DMEM) liquid medium, with 10\% fetal bovine serum (FBS) and $1 \%$ penicillin-streptomycin, at $37^{\circ} \mathrm{C}$, in an incubator supplied with $5 \% \mathrm{CO}_{2}$ and proper humidity. Cells were subcultured and the medium was changed 2-3 times a week.

2.5.2. Cytotoxicity and NO production. RAW 264.7 cells (5 $\times 10^{4}$ cells per well) were cultured in 96-well plate in DMEM containing $10 \%$ FBS for $24 \mathrm{~h}$ to become nearly confluent. Then cells were cultured with increasing concentrations of SV, SB, SS (125-500 $\mu \mathrm{g} \mathrm{mL}^{-1}$ ) in the presence of $100 \mathrm{ng} \mathrm{mL}{ }^{-1}$ LPS for $24 \mathrm{~h}$. After that, cells were incubated with $100 \mu \mathrm{L}$ of $0.5 \mathrm{mg} \mathrm{mL}^{-1}$ MTT for $4 \mathrm{~h}$ at $37^{\circ} \mathrm{C}$. After incubation, the colored formazan crystals formed in culture plate was dissolved in $0.04 \mathrm{~N} \mathrm{HCl}$ / isopropanol. The optical densities (OD) were measured at $570 \mathrm{~nm}$ using a microplate reader (Molecular Devices, USA). The viability of RAW 264.7 cells in each well was presented as compared with percentage of untreated control cells.

NO production was indirectly assessed by measuring the nitrite levels in the culture media using Griess reagent assay. Briefly, RAW 264.7 cells were seeded at a density of $5 \times 10^{4}$ cells per well in 96-well plates for $24 \mathrm{~h}$. After incubation, the cells were treated with SV, SB, SS $\left(125,250\right.$ and $\left.500 \mu \mathrm{g} \mathrm{mL}^{-1}\right)$ in the presence of LPS (100 $\mathrm{ng} \mathrm{mL}{ }^{-1}$ ) for $24 \mathrm{~h}$. The culture supernatant was collected for nitrite assay. Each of $100 \mu \mathrm{L}$ of culture media was mixed with an equal volume of Griess reagent (1\% sulfanilamide, $0.1 \%$ naphthyl ethylenediamine dihydrochloride and $5 \%$ phosphoric acid) and incubated at room temperature for $5 \mathrm{~min}$, the absorbance was measured at $540 \mathrm{~nm}$ with a microplate reader (Molecular Devices). Fresh culture media were used as blanks and the nitrite levels were determined by using a standard curve obtained from sodium nitrite.

2.5.3. Measurement of cytokines. Macrophages were seeded at $5 \times 104$ cells per well in 96-well plates. Cells were incubated with DMB $(62.5,125,250$ and $500 \mu \mathrm{M})$ in the presence of LPS (100 ng mL ${ }^{-1}$ ) for $24 \mathrm{~h}$. Cell culture supernatants were centrifuged at $5000 \times g$ for $3 \mathrm{~min}$ at $4{ }^{\circ} \mathrm{C}$ to remove insoluble material. Secreted IL-1â, IL-6 and TNF- $\alpha$ were measured in cell culture supernatants using commerciallyavailable ELISA kits (BioLegend, San Diego, CA) following the instructions provided by the manufacturers. The absorbance $(450 \mathrm{~nm})$ for each sample was analyzed using microplate reader and was interpolated with a standard curve. Results of three independent experiments were used for statistical analysis.

\subsection{Determination of anti-proliferative effect by human cell lines}

For the experiment, four human cancer cell lines were used. Two were lung cancer cell lines, human non-small cell lung cancer cell line A549 (purchased from the Bioresource Collection and Research Center) and human lung squamous carcinoma cell line $\mathrm{CH} 27$ (obtained from Professor Shih-lan Hsu, Taichung Veterans General Hospital); and two hepatocarcinoma cell lines, HepG2 and Hep3B (purchased from the Bioresource Collection and Research Center).

A549 cells were cultured in F-12 Nutrient mixture, containing $10 \%$ fetal bovine serum and 1\% penicillin-streptomycin. $\mathrm{CH} 27$ and HepG2 cells were cultured in DMEM, containing $10 \%$ FBS, 1\% penicillin-streptomycin, and $2 \mathrm{mM}$ L-glutamine. Hep3B cells were cultured in minimum essential medium (MEM), 
containing $10 \%$ FBS, $1 \%$ penicillin-streptomycin, $1 \%$ sodium pyruvate, and $1 \%$ non-essential amino acid.

\subsection{Statistical analysis}

Unless otherwise stated, all experiments were performed at least three times independently. Experimental results were presented as the mean \pm standard deviation (SD) of three parallel measurements. Statistical evaluation was carried out by one-way analysis of variance (ANOVA) followed by Scheffe's multiple range tests. Statistical significance was expressed as ${ }^{*} p<0$ : 05 , ${ }^{* *} p<0: 01$ and ${ }^{* * *} p<0: 001$.

\section{Results}

\subsection{Total polyphenol contents and antioxidant activities of crude extracts in vitro}

As shown in Table 1, the analysis of the phenolic levels in Sanghuang indicated that the extract at $500 \mu \mathrm{g} \mathrm{mL}^{-1}$ contained an equivalent of $135.5 \pm 1.8 \mu \mathrm{g} \mathrm{mg}^{-1}$ total polyphenol.

Table 1 shows the DPPH and ABTS radical scavenging activities of different Sanghuang varieties. The results indicated that the free radical scavenging ability of SS was significantly better than that of the other varieties $(p<0.05)$. SS exhibited the strongest antioxidant activities in scavenging DPPH and ABTS radicals, with $\mathrm{IC}_{50}$ values of $167.3 \pm 10.0 \mu \mathrm{g} \mathrm{mL} L^{-1}$ and $240.2 \pm$ $10.4 \mu \mathrm{g} \mathrm{mL}{ }^{-1}$, respectively.

\subsection{Analysis of chemical components and establishment of quality control}

Polyphenolic compounds are natural antioxidants that play major functional role in plants by scavenging free radicals. ${ }^{28}$ Polyphenolic compounds can be divided into two large categories: flavonoids and phenolic acids.

This study selected six types of phenolic acids found in Sanghuang, protocatechuic acid (1), protocatechualdehyde (2), caffeic acid (3), syringic acid (4), 2,5-dihydroxyterephtalic acid (DTA, 5), and 3,4-dihydroxybenzalacetone (DBL, 6), as reference compounds to qualitatively and quantitatively analyze the basidiocarp extracts from the three Sanghuang varieties.

HPLC analysis revealed the retention times (Rt) of protocatechuic acid, protocatechualdehyde, caffeic acid, and 3,4dihydroxybenzalacetone (DBL) to be $5.8,7.4,9.5$, and $16.0 \mathrm{~min}$, respectively, in case of all samples as shown in Fig. 1. The major absorption peaks of phenolic acids were consistent with the Photo Diode Array (PDA) spectra of the reference materials.

As shown in Table 2, the relative amounts of the four phenolic compounds found in SS were in the order of protocatechuic acid $\left(101.9 \pm 1.5 \mu \mathrm{g} \mathrm{mg}{ }^{-1}\right.$ extract $)>$ protocatechualdehyde $\left(51.9 \pm 0.1 \quad \mu \mathrm{g} \quad \mathrm{mg}^{-1}\right.$ extract $)>3,4-$ dihydroxybenzalacetone (DBL) $\left(48.6 \pm 1.5 \mu \mathrm{g} \mathrm{mg}{ }^{-1}\right.$ extract $)>$ caffeic acid (19.1 $\pm 0.1 \mu \mathrm{g} \mathrm{mg}^{-1}$ extract), respectively.

Table 2 shows the relative amounts of the hispidin derivative compounds found in SS were in the order of inoscavin C (46.9 \pm $3.3 \mu \mathrm{g} \mathrm{mg}^{-1}$ extract $)>$ inoscavin A $\left(17.0 \pm 2.4 \mu \mathrm{g} \mathrm{mg}^{-1}\right.$ extract $)$, respectively.

\subsection{Assessment of anti-inflammatory activity}

The RAW 264.7 cells were treated with different concentrations of the extracts. After $24 \mathrm{~h}$, MTT assay was performed to analyze the degree of cytotoxicity of the extracts. As evident from Fig. 2, neither of the three extracts exhibited any major cytotoxic effect nor did they significantly affect the normal growth of RAW 264.7 cells even at the concentration of $500 \mu \mathrm{g} \mathrm{mL}^{-1}$. This suggested that the decreased levels of pro-inflammatory mediators in subsequent experiments were not due to decreased number of cells.

To elucidate whether Sanghuang extracts could inhibit lipopolysaccharide (LPS)-induced pro-inflammation in RAW 264.7 cells, we assessed the level of pro-inflammatory markers nitric oxide (NO), tumor necrosis factor (TNF- $\alpha$ ), interleukin (IL)-1 $\beta$, and IL-6. As shown in Fig. 2, RAW 264.7 cell viability under different doses of SS was not affected. Concurrent application of non-toxic doses of SS significantly reduced the amounts of LPS-induce NO and pro-inflammatory cytokines produced in RAW 264.7 cells. Other groups have demonstrated similar results. Fig. 2 shows the results of the anti-inflammatory ability of Sanghuang extracts from the three varieties. Quite evidently, SS had a significantly greater ability to inhibit the synthesis of NO and pro-inflammatory cytokines than other varieties $(p<0.05)$. We also found that SS could significantly decrease the expression levels of iNOS and COX-2 as shown in Fig. 3.

\subsection{Assessment of tumor cell growth inhibitory ability}

Liver cancer cells (HepG2, Hep3B) and lung cancer cells (A549, $\mathrm{CH} 27$ ) were treated with $500 \mu \mathrm{g} \mathrm{mL} \mathrm{L}^{-1}$ of Sanghuang basidiocarp ethanol extracts from two similar Sanghuangs varieties and

Table 1 Total phenolic contents and radical scavenging activity of ethanol extracts from three Sanghuang species (SV, SB and SS) $\#, a$

\begin{tabular}{llll}
\hline Samples & Total phenols $(\mu \mathrm{g} \mathrm{GE} \mathrm{mg})^{\dagger}$ & DPPH scavenging activity $\left(\mathrm{IC}_{50}, \mu \mathrm{g} \mathrm{mL}{ }^{-1}\right)$ & $\mathrm{ABTS}^{\dagger}$ scavenging activity $\left(\mathrm{IC}_{50}, \mu g \mathrm{~mL}^{-1}\right)$ \\
\hline SV & $126.9 \pm 10.8$ & $210.3 \pm 22.5^{* *, \mathrm{~b}}$ & $742.0 \pm 41.2^{* * *, \mathrm{c}}$ \\
SB & $132.5 \pm 11.6$ & $180.2 \pm 9.4^{\mathrm{a}}$ & $529.1 \pm 41.6^{* * *, \mathrm{~b}}$ \\
SS & $135.5 \pm 1.8$ & $167.3 \pm 10.0^{\mathrm{a}}$ & $240.2 \pm 10.4^{\mathrm{a}}$
\end{tabular}

$a{ }^{*}$ Values are expressed as mean \pm S.D. of three replicates. ${ }^{\dagger}$ Data expressed in $\mu$ g gallic acid equivalent per mg extract weight $\left(\mu \mathrm{g}\right.$ GE mg $\left.{ }^{-1}\right) . *^{* *} p<$ 0.01 , and ${ }^{* *} p<0.001$, were compared with sample of SS group (one-way ANOVA followed by Scheffe's multiple range tests). Means with different letters represent significantly different $(p<0.05)$ by Scheffé's test. 

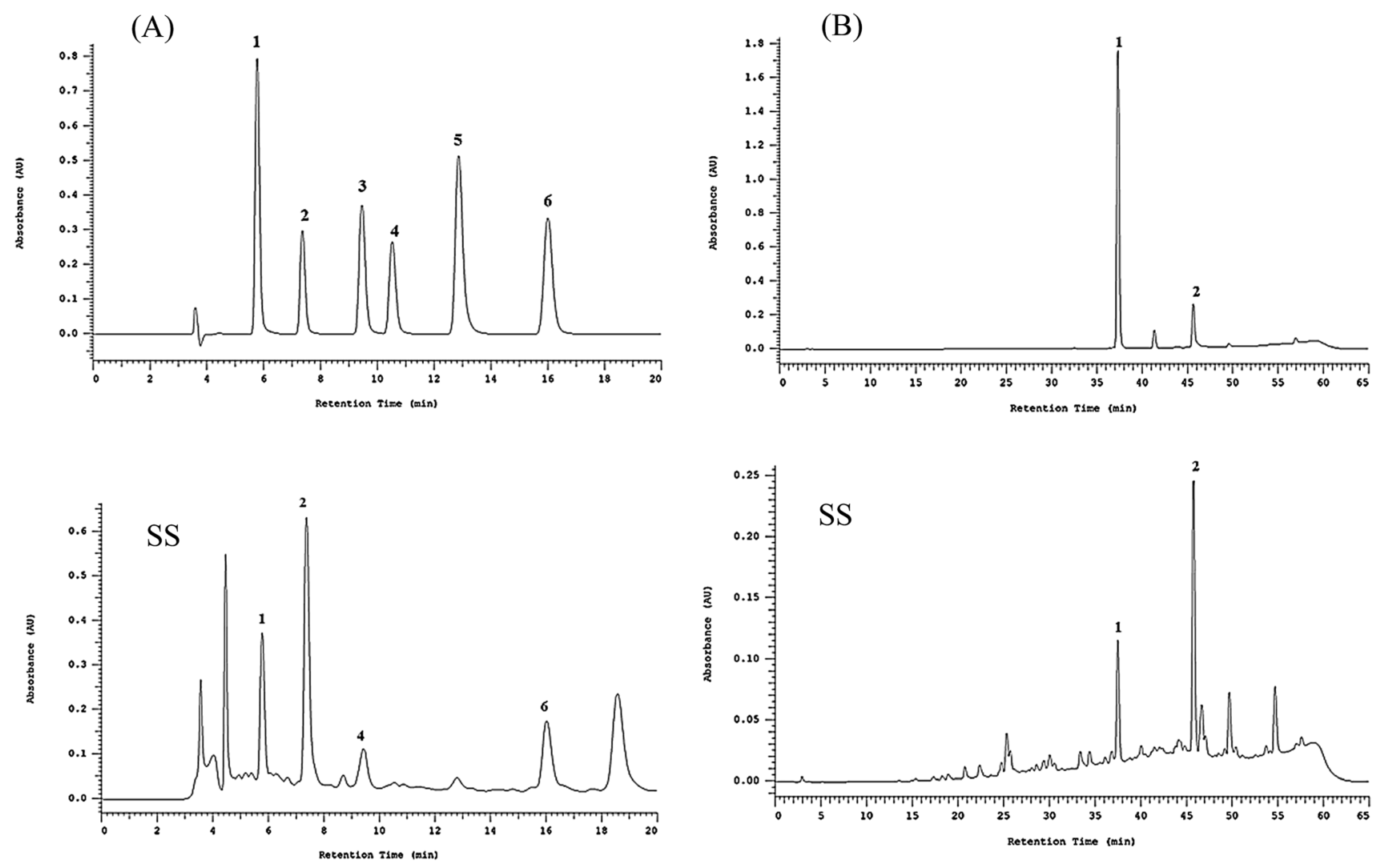

Fig. 1 HPLC chromatogram of 70\% ethanol extracts of Sanghuang (SS). Determination of phenolic compounds (A): the peaks indicate the following: protocatechuic acid (1), protocatechualdehyde (2), caffeic acid (3), syringic acid (4), 2,5-dihydroxyterephtalic acid or DTA (5), and 3,4dihydroxybenzalacetone or DBL (6). Hispidin derivative compounds (B): the peaks indicate the following: inoscavin A (1), and inoscavin C (2).

Table 2 Quantification of identified individual phenolic compounds and hispidin derivatives using HPLC-DAD system ${ }^{a}$ $\underline{\text { Sample }\left(\mu \mathrm{g} \mathrm{mg}^{-1}\right)}$

Standards Residence time (min)

SV

SB

SS

\section{(A) Phenolic compounds}

Protocatechuic acid

Protocatechualdehyde

5.8
7.4
9.5
10.5
12.9
16.0

$41.5 \pm 0.6$

$8.5 \pm 0.2$

$81.9 \pm 1.4$

$42.5 \pm 0.8$

$101.9 \pm 1.5$

Caffeic acid

$-$

Syringic acid

DTA (2,5-dihydroxyterephtalic acid)

-

DBL (3,4-dihydroxybenzalacetone)

16.0

$-$

$9.5 \pm 0.1$

-

$-$

$8.8 \pm 0.4$

$51.9 \pm 0.1$

$19.1 \pm 0.1$

(B) Hispidin derivative compounds

Inoscavin A

37.3

Inoscavin $\mathrm{C}$

45.7

-

$25.2 \pm 4.2$

$30.6 \pm 0.4$

$17.0 \pm 2.4$

${ }^{a}$ Values are expressed as mean \pm S.D. of three replicates.

Sanghuangs for $48 \mathrm{~h}$, followed by MTT assay to assess its growth inhibitory ability in cancer cells. As shown in Fig. 4, with the cell viability of the control group taken as $100 \%$, extracts from different Sanghuang basidiocarps showed significant growth inhibitory effects in cancer cells to different degrees. In liver cancer cells HepG2 and Hep3B treated with SS, the rates of tumor cell growth inhibition were $53.3 \pm 1.9 \%$ and $35.6 \pm 6.6 \%$, respectively, while it was $79.2 \pm 3.4 \%$ and $53.2 \pm 8.4 \%$, in lung cancer cells A549 and $\mathrm{CH} 27$, respectively. Other groups have demonstrated similar results.

The tumor cell growth inhibitory ability of the different Sanghuang extracts is evident in Fig. 4. While all three varieties exhibited tumor growth suppressive effect, the inhibitory ability of SS was superior to that of other Sanghuang varieties $(p<0.05)$. 

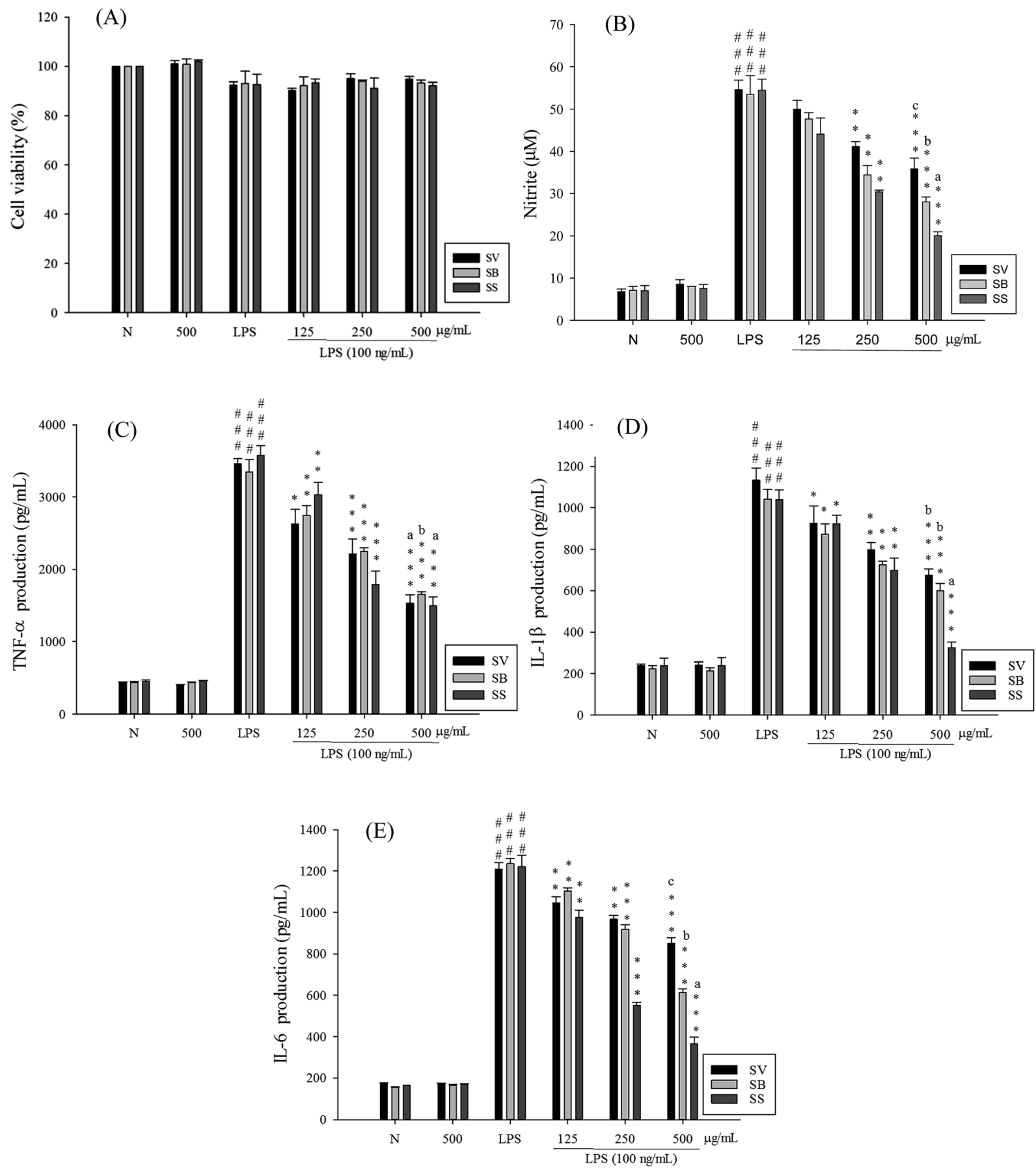

Fig. 2 Three Sanghuang species (SV, SB and SS) inhibited LPS-induced cell inflammation in RAW 264.7 cells. Cytotoxicity (A) of SV, SB and SS in LPS-stimulated RAW 264.7 cells. Cells were treated with SV, SB and SS at 125,250 and $500 \mu \mathrm{g} \mathrm{mL}^{-1}$ for $24 \mathrm{~h}$, and cell viability was assayed by the MTT assay. NO (B), TNF- $\alpha$ (C), IL-1 $\beta$ (D), and IL-6 (E) production in LPS-stimulated RAW 264.7 cells. Cells were incubated with or without LPS (100 $\mathrm{ng} \mathrm{m \textrm {m } ^ { - 1 }}$ ) in the presence of various doses $\left(125,250\right.$ and $500 \mu \mathrm{g} \mathrm{mL}^{-1}$ ) of SV, SB and SS for $24 \mathrm{~h}$. Values are expressed as mean \pm S.D. of three

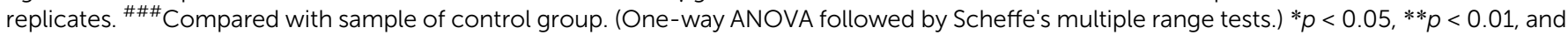
$* * * p<0.001$, were compared with LPS-alone group. Means with different letters represent significantly different $(p<0.05)$ by Scheffe's test.

\section{Discussion}

Sanghuang is known as a species of medicinal fungi. There are very few numbers of authentic wild mulberry Sanghuang; however, there are diverse species of multiporous fungi that are similar to Sanghuang. Authentic Sanghuang has only been confirmed by Wu et al. in 2012, and it was published as a new species named Inonotus sanghuang. ${ }^{19}$ Further studies of the microscopic structure of Sanghuang and molecular systematics revealed that Sanghuang and similar species did not belong to the genus Inonotus; in 2015, Sanghuang and similar species were published and classified as a new genus: Sanghuangporus. 

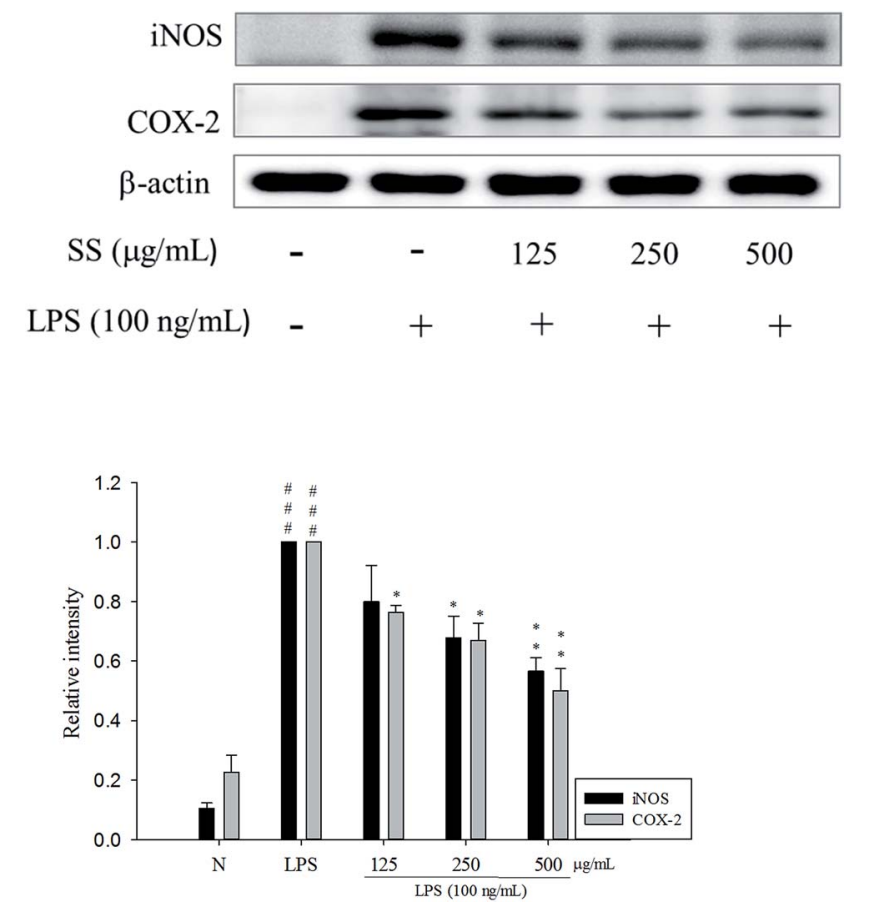

Fig. 3 Effects of SS on iNOS and COX-2 protein expression in LPSinduced RAW 264.7 cells. Cells were incubated with or without LPS (100 $\mathrm{ng} \mathrm{mL}^{-1}$ ) in the presence of various concentrations $(125,250$ and $500 \mu \mathrm{g} \mathrm{mL}^{-1}$ ) of SS for $24 \mathrm{~h}$. The data were presented as mean $\pm \mathrm{SD}$ for

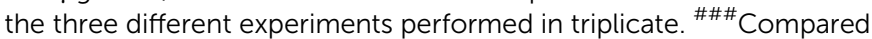
with sample of control group (one-way ANOVA followed by Scheffe's multiple range tests). $* * p<0.01$, and $* * * p<0.001$ were compared with LPS-alone group

The scientific name of Sanghuang was later changed to Sanghuangporus sanghuang. ${ }^{20}$

Biological oxidations are necessary to generate energy for cellular processes in living organisms. Free radicals such as reactive oxygen species, generated during biological oxidations, cause lipid peroxidation, which is associated with various human pathologies including cancer, inflammation, and ageing. ${ }^{29} \mathrm{~A}$ basal level of free radicals is generated during normal metabolism, which can be counteracted by the body's indigenous antioxidant defense mechanisms, thus maintaining a state of dynamic equilibrium. Antioxidants are used to slow down the production of reactive oxygen species and free radicals. The currently known naturally available antioxidants include lipoic acid; vitamins $\mathrm{A}, \mathrm{C}$, and E; glutathione; and coenzyme Q10. ${ }^{30}$ Plant secondary metabolites, such as flavonoids, polyphenols, and carotenoids, have also been shown to have antioxidant effects. ${ }^{28}$ Our results showed that $S$. sanghuang had good DPPH and ABTS scavenging ability, and was rich in polyphenolic compounds. Research has shown that plant polyphenols are strong antioxidants owing to the presence of $\mathrm{OH}$ group in their structures, which is capable of capturing free radicals and reducing the oxidative damage to proteins, lipids and DNA, thereby preventing the onset of various diseases. The HPLC analysis results of this study suggested that $S$. sanghuang contained abundant polyphenolic components, and the principal phenolic acids were protocatechuic acid and protocatechualdehyde, followed by caffeic acid and 3,4-dihydroxybenzalacetone (DBL). Therefore, we believe that S. sanghuang is similar to T. linteus, and can attenuate the production of reactive oxygen species (ROS) and free radicals via its high antioxidant capacity, mediated by polyphenolic compounds that serve as antioxidants.

Inflammation is a non-specific reaction of the immune system induced by infection or tissue damage. It mainly involves release of nitric oxide and pro-inflammatory cytokines, such as TNF $\alpha$, IL-1 and IL-6, by activated macrophages and induction of leukocyte infiltration in the infected region, which results in the development of four cardinal symptoms: redness, swelling, heat, and pain. ${ }^{12,31}$ Inflammation is a self-protective reaction of the body by activating the immune system and killing malignant tumor cells. However, excess or persistent inflammation is associated with the pathogenesis of various diseases. $^{31,32}$ NO and pro-inflammatory cytokines, secreted by activated macrophages, play an important role during the inflammatory response and are used for the detection and quantification of inflammation. ${ }^{32}$

It has been reported that extracts of T. linteus and $S$. baumii, which have been mistakenly applied as Sanghuangporus species in the past, exhibit a good anti-inflammatory effect in LPSinduced mouse macrophages, and can inhibit the activation of NF- $\mathrm{B}$ and MAPKs pathways to exert an anti-inflammatory effect. $^{21,23}$ On the other hand, our experimental results indicated that $S$. sanghuang can inhibit the expression of iNOS and COX-2, and can attenuate the production of pro-inflammatory cytokines. It has a better anti-inflammatory capacity than either $S$. baumii or $S$. vaninii. However, extracts of $T$. linteus have also been reported to exhibit a good anti-inflammatory effect in a mouse model of intestinal inflammation..$^{33}$ Therefore, we believe that the anti-inflammatory capacity of $S$. sanghuang, which is now classified as a Sanghuang species, can be used in the future to investigate more diseases caused by inflammation, such as sepsis and acute lung injury, by using animal models.

In recent years, cancer has been one of the main causes of death worldwide. Thus, topics of cancer research, such as pathological mechanism, treatment methods, and anti-cancer drug research and development, have been widely studied in the past two decades. In Taiwan, since 1982, cancer has ranked first in the top ten causes of death for 24 consecutive years. Cancer treatments mainly include chemotherapy, radiotherapy, and immunotherapy. ${ }^{34}$ However, these treatment methods have side effects on the body, and therefore, the inhibitory effect of natural plant extracts (taxol, curcumin) on cancer is worth further exploration. ${ }^{18}$

Furthermore, extracts of $T$. linteus and S. baumii, which have been mistakenly applied as $S$. sanghuang in the past, have been reported to be used to inhibit the growth of multiple types of cancer cells, including SK-Hep-1, Hep3B, HepG2, and A549 cells. ${ }^{23}$ Our experimental results indicated that $S$. sanghuang can also inhibit the growth of lung and liver cancer cells with a better inhibitory capacity than either $S$. baumii or $S$. vaninii. Hence, the possible mechanism of cancer inhibition by $S$. sanghuang can be further explored in the future to develop natural anti-cancer drugs. 

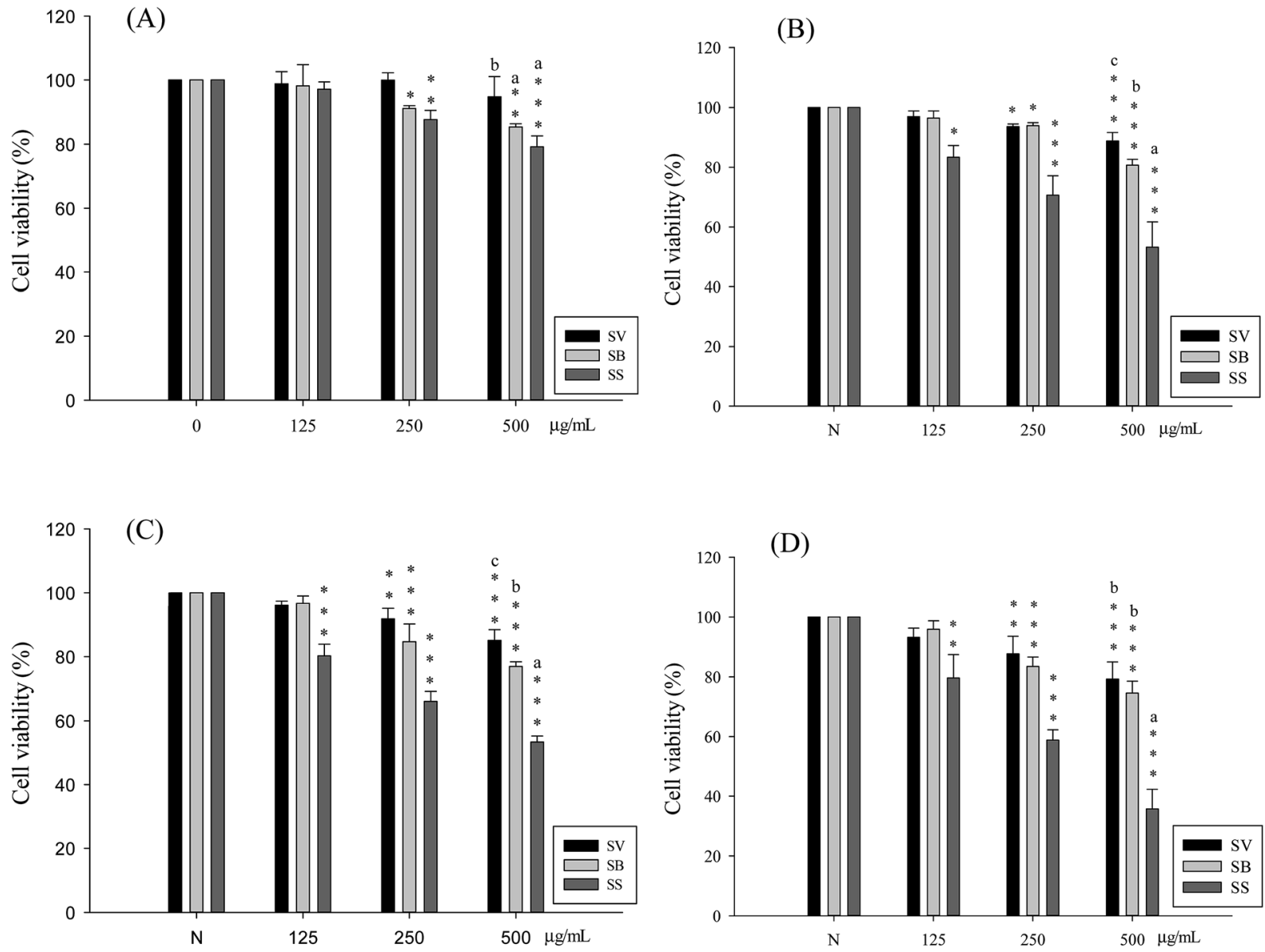

Fig. 4 Different concentrations of three Sanghuang species (SV, SB and SS) had varied effects on the A549 cell (A), CH27 cell (B), HepG2 cell (C) and Hep3b cell (D) growth survival rate. Values are expressed as mean \pm S.D. of three replicates. $* p<0.05, * * p<0.01$, and $* * * p<0.001$, compared with sample of control group (one-way ANOVA followed by Scheffe's multiple range tests). Means with different letters represent significantly different $(p<0.05)$ by Scheffe's test.

\section{Conclusion}

In conclusion, we found all Sanghuang basidiocarps varieties to have anti-proliferative effects on hepatic and lung carcinoma cells. Additionally, I. sanghuang showed the strongest inhibitory effects on LPS-induced NO production in RAW 264.7 cells. Overall, these results suggest that Sanghuang is a promising antiinflammatory agent that suppresses iNOS and COX-2 expression. Among the three varieties tested, I. sanghuang was found to be the most effective variety with a potential for development into dietary supplement and/or a natural herbal medicine.

\section{Conflict of interest}

The authors have no conflicts of interest.

\section{Acknowledgements}

The authors want to thank the financial supports from the National Science Council (MOST 103-2320-B-468-002 and MOST 105-2320-B-039-046), China Medical University (CMU) (CMU103-ASIA-22, ASIA104-CMUH-06 and CMU105-ASIA-23), and this study also is supported in part by Taiwan Ministry of Health and Welfare Clinical Trial and Research Center of Excellence (MOHW105-TDU-B-212-133019).

\section{References}

1 L. A. Pham-Huy, H. He and C. Pham-Huy, Free radicals, antioxidants in disease and health, Int. J. Biomed. Sci., 2008, 4(2), 89-96.

2 A. Rahal, A. Kumar, V. Singh, B. Yadav, R. Tiwari, S. Chakraborty and K. Dhama, Oxidative stress, prooxidants, and antioxidants: the interplay, BioMed Res. Int., 2014, 761264.

3 K. Rahman, Studies on free radicals, antioxidants, and co-factors, Clin. Interventions Aging, 2007, 2(2), 219-236.

4 L. C. Wu, A. F. Jou, S. H. Chen, C. Y. Tien, C. F. Cheng, N. C. Fan and J. A. Ho, Antioxidant, anti-inflammatory and anti-browning activities of hot water extracts of oriental herbal teas, Food Funct., 2010, 1(2), 200-208.

5 M. Liu, X. Q. Li, C. Weber, C. Y. Lee, J. Brown and R. H. Liu, Antioxidant and antiproliferative activities of raspberries, J. Agric. Food Chem., 2002, 50(10), 2926-2930. 
$6 \mathrm{~K}$. Wolfe, X. Wu and R. H. Liu, Antioxidant activity of apple peels, J. Agric. Food Chem., 2003, 51(3), 609-614.

7 L. C. Wu, J. A. A. Ho, M. C. Shieh and I. W. Lu, Antioxidant and antiproliferative activities of spirulina and shlorella water extracts, J. Agric. Food Chem., 2005, 53(10), 4207-4212. 8 G. J. Huang, J. S. Deng, C. C. Chen, C. J. Huang, P. J. Sung, S. S. Huang and Y. H. Kuo, Methanol extract of Antrodia camphorata protects against lipopolysaccharide-induced acute lung injury by suppressing NF- $\mathrm{B}$ and MAPK pathways in mice, J. Agric. Food Chem., 2014, 62, 5321-5329.

9 J. S. Deng, S. S. Huang, T. H. Lin, M. M. Lee, C. C. Kuo, P. J. Sung, W. C. Hou, G. J. Huang and Y. H. Kuo, The analgesic and anti-inflammatory bioactivities of eburicoic acid and dehydroeburicoic acid isolated from Antrodia camphorata on the inflammatory mediator expression in mice, J. Agric. Food Chem., 2013, 61, 5064-5071.

10 A. Mantovani, P. Allavena, A. Sica and F. Balkwill, Cancerrelated inflammation, Nature, 2008, 454(7203), 436-444.

11 P. H. Shie, S. Y. Wang, H. L. Lay and G. J. Huang, 4,7Dimethoxy-5-methyl-1,3-benzodioxole from Antrodia camphorata inhibits LPS-induced inflammation via suppression of NF- $\kappa \mathrm{B}$ and induction HO-1 in RAW264.7 cells, Int. Immunopharmacol., 2016, 31, 186-194.

12 S. Marzocco, L. Calabrone, S. Adesso, M. Larocca, S. Franceschelli, G. Autore, G. Martelli and R. Rossano, Anti-inflammatory activity of horseradish (Armoracia rusticana) root extracts in LPS-stimulated macrophages, Food Funct., 2015, 6(12), 3778-3788.

13 K. C. Li, Y. L. Ho, G. J. Huang and Y. S. Chang, Anti-oxidative and anti-inflammatory effects of Lobelia chinensis in vitro and in vivo, Am. J. Chin. Med., 2015, 43(2), 269-287.

14 P. H. Shie, S. S. Huang, J. S. Deng and G. J. Huang, Spiranthes sinensis suppresses production of pro-inflammatory mediators by down-regulating the NF- $\kappa \mathrm{B}$ signaling pathway and up-regulating $\mathrm{HO}-1 / \mathrm{Nrf} 2$ anti-oxidant protein, Am. J. Chin. Med., 2015, 43, 969-989.

15 M. Hassan, H. Watari, A. AbuAlmaaty, Y. Ohba and N. Sakuragi, Apoptosis and molecular targeting therapy in cancer, BioMed Res. Int., 2014, 2014, 150845.

16 E. Safarzadeh, S. Sandoghchian Shotorbani and B. Baradaran, Herbal medicine as inducers of apoptosis in cancer treatment, Adv. Pharm. Bull., 2014, 421-427.

17 S. Y. Yin, W. C. Wei, F. Y. Jian and N. S. Yang, Therapeutic applications of herbal medicines for cancer patients, $J$. Evidence-Based Complementary Altern. Med., 2013, 302426.

18 M. Anwar, S. Akhter, N. Mallick, S. Mohapatra, S. Zafar, M. M. Rizvi, A. Ali and F. J. Ahmad, Enhanced anti-tumor efficacy of paclitaxel with PEGylated lipidic nanocapsules in presence of curcumin and poloxamer: in vitro and in vivo studies, Pharmacol. Res., 2016, 113, 146-165.

19 S. H. Wu, Y. C. Dai, T. Hattori, T. W. Yu, D. M. Wang, E. Parmasto, H. Y. Chang and S. Y. Shin, Species clarification for the medicinally valuable sanghuang mushroom, Bot. Stud., 2012, 53, 135-149.

20 L. W. Zhou, J. Vlasák, C. Decock, A. Assefa, J. Stenlid, D. Abate, S. H. Wu and Y. C. Dai, Global diversity and taxonomy of the Inonotus linteus complex (Hymenochaetales, Basidiomycota):
Sanghuangporus gen. nov., Tropicoporus excentrodendri and T. guanacastensis gen. et spp. nov., and 17 new combinations, Fungal Divers., 2016, 77, 335-347.

21 H. Chen, T. Tian, H. Miao and Y. Y. Zhao, Traditional uses, fermentation, phytochemistry and pharmacology of Phellinus linteus: a review, Fitoterapia, 2016, 113, 6-26.

22 Y. C. Chen, H. Y. Chang, J. S. Deng, J. J. Chen, S. S. Huang, I. H. Lin, W. L. Kuo, W. Chao and G. J. Huang, Hispolon from Phellinus linteus induces G0/G1 cell cycle arrest and apoptosis in NB4 human leukaemia cells, Am. J. Chin. Med., 2013, 41, 1439-1457.

23 G. J. Huang, S. S. Huang and J. S. Deng, Anti-inflammatory activities of inotilone from Phellinus linteus through the inhibition of MMP-9, NF- $\mathrm{B}$, and MAPK activation in vitro and in vivo, PLoS One, 2012, 7, e35922.

24 S. H. Kim, H. S. Lee, S. Lee, J. Cho, K. Ze, J. Sung and Y. C. Kim, Mycelial culture of Phellinus linteus protectsprimary cultured rat hepatocytes against hepatotoxins, J. Ethnopharmacol., 2004, 95, 367-372.

25 P. W. Hsieh, J. B. Wu and Y. C. Wu, Chemistry and biology of Phellinus linteus, Biomedicine, 2013, 106-113.

26 J. G. Han, M. W. Hyun, C. S. Kim, J. W. Jo, J. H. Cho, K. H. Lee, W. S. Kong, S. K. Han, J. Oh and G. H. Sung, Species identity of Phellinus linteus (sanghuang) extensively used as a medicinal mushroom in Korea, J. Microbiol., 2016, 54(4), 290-295.

27 S. S. Huang, C. S. Chiu, T. H. Lin, M. M. Lee, C. Y. Lee, S. J. Chang, W. C. Hou, G. J. Huang and J. S. Deng, Antioxidant and anti-inflammatory activities of aqueous extract of Centipeda minima, J. Ethnopharmacol., 2013, 147(2), 395-405.

28 D. M. Kasote, S. S. Katyare, M. V. Hegde and H. Bae, Significance of antioxidant potential of plants and its relevance to therapeutic applications, Int. J. Biol. Sci., 2015, 11(8), 982-991.

29 S. Reuter, S. C. Gupta, M. M. Chaturvedi and B. B. Aggarwal, Oxidative stress, inflammation, and cancer: how are they linked?, Free Radical Biol. Med., 2010, 49(11), 1603-1616.

30 E. C. Gomes, A. N. Silva and M. R. de Oliveira, Oxidants, antioxidants, and the beneficial roles of exercise-induced production of reactive species, Oxid. Med. Cell. Longevity, 2012, 756132.

31 J. Lee, S. J. Ha, H. J. Lee, M. J. Kim, J. H. Kim, Y. T. Kim, K. M. Song, Y. J. Kim, H. K. Kim and S. K. Jung, Protective effect of Tremella fuciformis Berk extract on LPS-induced acute inflammation via inhibition of the NF- $\kappa$ B and MAPK pathways, Food Funct., 2016, 7(7), 3263-3272.

32 H. Lv, W. Ren, Y. Zheng, L. Wang, G. Lu, P. Yi and X. Ci, Tenuigenin exhibits anti-inflammatory activity via inhibiting MAPK and NF- $\mathrm{B}$ and inducing Nrf2/HO-1 signaling in macrophages, Food Funct., 2016, 7(1), 355-363.

33 M. Song and H. J. Park, Anti-inflammatory effect of Phellinus linteus grown on germinated brown rice on dextran sodium sulfate-induced acute colitis in mice and LPS-activated macrophages, J. Ethnopharmacol., 2014, 154(2), 311-318.

34 C. J. Chen, S. L. You, L. H. Lin, W. L. Hsu and Y. W. Yang, Cancer epidemiology and control in Taiwan: a brief review, Jpn. J. Clin. Oncol., 2002, 32, S66-S81. 